\section{Das „tonangebende“ Werk musikerspezifischer Erkrankungen}

$\mathrm{M}$ usizieren macht Freude und hat nachweislich eine positive Wirkung auf unsere Gesundheit. Mit zunehmender Professionalisierung wachsen jedoch die körperlichen und mentalen Anforderungen. Nicht zuletzt aufgrund der enormen Leistungsansprüche benötigt ein großer Teil der professionellen Sänger und Instrumentalisten eine fundierte medizinische Betreuung.

Die Musikermedizin, ursprünglich in den USA entstanden und inzwischen auch in Deutschland zunehmend etabliert, berücksichtigt das gesamte Spektrum musikerspezifischer Erkrankungen. Bedingt durch die vielfältigen Anforderungen beim Instru- mentalspiel und Singen sind dabei verschiedene Körperregionen betroffen, auch psychische Probleme sind eine wesentliche Manifestation. Neben speziellen Diagnostik- und Behandlungsmodellen stehen bei der Musikermedizin besonders die Prävention und Gesundheitsförderung im Fokus.

Die „MusikerMedizin“ schafft kompetent die Voraussetzungen, um musikerspezifische Probleme erkennen und behandeln zu können. Grundlagenkenntnisse werden didaktisch hervorragend vermittelt, spezielles Wissen anhand von Einzelfallbeispielen eindrücklich präsentiert. Ärzte und Therapeuten, die mit den

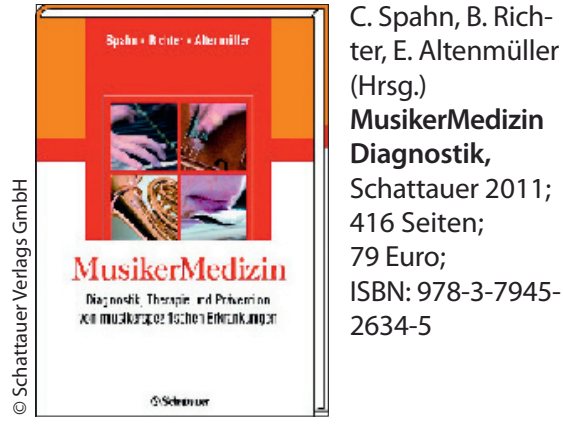

spezifischen Beschwerden von Sängern und Instrumentalisten konfrontiert sind, finden hier umfassendes Wissen und Handlungsempfehlungen zu allen medizinischen Aspekten - praxisnah und multidisziplinär. Prof. Dr. Klaus A. Milachowski

\title{
Unfallbegutachtung: Für HNO-Ärzte keine neuen Erkenntnisse
}

\begin{abstract}
A uch in der 12. Auflage der „Unfallbegutachtung" wollen die Autoren Friedrich Mehrhoff, Renate Chr. Meindl und Gert Muhr die traditionellen „Brücken" im Grenzbereich zwischen rechtlichem und medizinischem Sachverstand bauen.

Für einen HNO-Gutachter bringt die Neuauflage jedoch keine neuen Erkenntnisse. Insbesondere ist der medizinische Bereich unverändert geblieben. Das Fachgebiet Hals-Nasen-Ohren wird auch
\end{abstract}

nur ganz peripher auf fünf Seiten erwähnt und ist außerdem nicht ganz korrekt.

Das neue Recht der gesetzlichen Unfallversicherung und die neuen Rechtsgrundlagen und Begrifflichkeiten im sozialen Entschädigungsrecht haben die Autoren zwar in dieser Auflage integriert, sie können jedoch genauso gut auch den gängigen Gutachtensbüchern, die der Hals-Nasen-Ohren-Arzt benötigt, entnommen werden. Die 12. Auflage

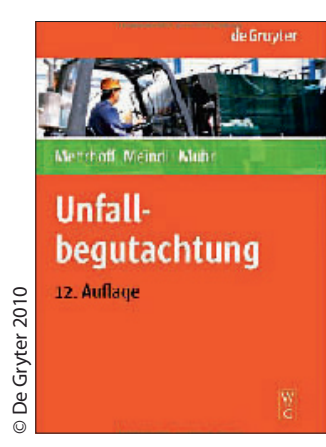

F. Mehrhoff, R. Ch. Meindl, G. Muhr Unfallbegutachtung De Gruyter 2010 12. Auflage; 384 Seiten; 79,95 Euro; ISBN 978-3-11020230-4

der Unfallbegutachtung kann einem HNO-ärztlichen Gutachter nicht empfohlen werden.

Prof. Dr. Karin Schorn

\section{Sicher verordnen bei älteren Patienten}

$\mathrm{M}$ enschen im Alter über 65 Jahren bilden die am stärksten wachsende Bevölkerungsgruppe und stellen einen Großteil der Patienten in den Arztpraxen. Ältere Patienten haben häufig mehrere - oft chronische - Erkrankungen. Wegen der Vielfachmedikation mit kaum vorhersehbaren Interaktionen, geänderter biologischer Abläufe wie verminderter Leberdurchblutung und Nierenfunktion und nicht zuletzt auch mangelnder Compliance kommt es bei diesen Patienten über- durchschnittlich oft zu unerwünschten Arzneimittelwirkungen.

Martin Wehling und Heinrich Burkhardt beantworten in ihrem Buch „Arzneitherapie für Ältere“ wichtige Fragen des Alltags in der Praxis wie: Welche Besonderheiten gibt es? Welche Nebenwirkungen und Wechselwirkungen müssen besonders beachtet werden und können unter Umständen als neue eigenständige Erkrankungen fehlinterpretiert werden? Welche Arzneimittel sind für ältere $\mathrm{Pa}$ -

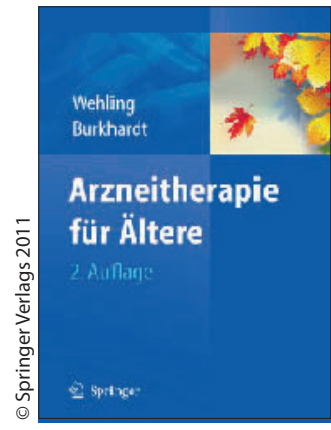

M. Wehling, H. Burkhardt Arzneitherapie für Ältere Springer Verlag 2011, 2. Auflage; 284 Seiten; 49,95 Euro; ISBN 978-3-64217307-3

tienten ungeeignet bzw. erfordern besondere Vorsicht? 